\title{
Age-Related Maturation of Wave V Latency of Auditory Brainstem Response in Children
}

\author{
Mukesh Sharma ${ }^{1,2}$, Sampan Singh Bist ${ }^{2}$, and Santosh Kumar ${ }^{1,2}$ \\ ${ }^{1}$ Rehabilitation Unit of Speech and Hearing, ${ }^{2}$ Department of ENT, Himalayan Institute of Medical Sciences, SRHU, Jollygrant, \\ Dehradun, India
}

Received April 22, 2016

Revised June 19,2016

Accepted August 8, 2016

Address for correspondence
Mukesh Sharma, MASLP
Rehabilitation unit
of Speech and Hearing,
Department of ENT,
Himalayan Institute
of Medical Sciences, SRHU,
Jollygrant, Dehradun-248016, India
Tel +91-9012848575
Fax +91-1352471157
E-mail mukeshaslp@gmail.com

Background and Objectives: Auditory brainstem response (ABR) is a noninvasive measurement of a stimulus-locked, synchronous electrical event. ABR provides information concerning the functional integrity of brainstem nuclei. Age is a key factor in the interpretation of $A B R$ peak latency among different age groups. Progressively with time it follows a "maturation pattern" during which latencies decrease. Wave $\mathrm{V}$ is very prominent and reliable for detection of threshold in children. The present study was performed to see the effect of age related auditory maturation on ABR wave $V$ latency in children. Subjects and Methods: The study involved 80 subjects ranging in age from birth to 12 years. The subjects were divided equally into eight age groups. ABR were elicited by an acoustic click stimuli, brainstem responses collected through electrode and recorded at the same time. Latency of wave $\mathrm{V}$ was acknowledged. Results: Wave $\mathrm{V}$ latency decreased rapidly in early childhood, became slower after 3 years of age and completely matured by 12 years of age. There was no significant difference in latency of wave $\mathrm{V}$ between the ears with age. Conclusions: There is a distinct maturation pattern of wave $V$ latency in ABR for both ears. ABR is a reliable test to assess the functional maturation of wave $\mathrm{V}$ in children.

J Audiol Otol 2016;20(2):97-101

\section{Introduction}

Auditory brainstem response (ABR) is a noninvasive measure of far-field representation of stimulus-locked and synchronous electrical events. It is a measure of electrical potential at the scalp in response to an acoustic signal, which provides information concerning the functional integrity of brainstem nuclei. The inferior colliculus is the primary relay center between ascending projections from the lower brainstem nuclei to the thalamus. The wave $\mathrm{V}$ is generated primarily within the inferior colliculus [1]. Wave V is the most robust ABR parameter for detection of threshold. During the first 2 years of life, peak latencies become progressively earlier and peak amplitude increases [1]. The auditory pathway of the human brainstem from the proximal end of the cochle-

This is an Open Access article distributed under the terms of the Creative Commons Attribution Non-Commercial License (http://creativecommons.org/licenses/by-nc/3.0/) which permits unrestricted non-commercial use, distribution, and reproduction in any medium, provided the original work is properly cited. ar nerve to the inferior colliculus undergoes myelination between fetal week 26 and 29. The axon of the cochlear nerve and brainstem pathways acquire linear arrays of oligodendrocytes and myelin sheaths by week 26 of gestation. Myelination in all auditory pathways occurs in week 29. Myelination density increases in all pathways until at least 1 year postnatal age. Time of onset of myelination coincides with the onset of acoustic motor reflexes and brainstem auditory evoked responses, processes which depend on the rapid and synchronized conduction of auditory impulses in the cochlear nerve and brainstem [2]. Increase in myelin density is a key factor in the steady decrease in ABR II-V latency. ABR latency changes occur because of the rapid increase in axonal myelin density in the cochlear nerve and brainstem pathways [1]. Weber [3] conducted a study on 130 infants to develop wave $\mathrm{V}$ latency norms and also for wave III latency of ABR for different conceptual age. Additional norms were also developed using ABR inter-wave intervals (I-III, III-V) as an indicator of brain stem maturation. The results suggest- 
ed that wave III and wave I-III inter-wave interval are more sensitive than wave $\mathrm{V}$ for auditory brainstem maturation. The same study also reported that conceptual age is not as satisfactory as the inter-wave-interval. Jackobson and Morehouse [4] examined the ABR of 125 normal ears in subjects 40-49 weeks in gestational age, with unfiltered clicks used as stimuli. The absolute latencies were prolonged in all age groups and decreased as gestational age increased, with the exception of wave I, which approximated adult latency by 2 months of age. The authors recorded a mean latency shift of $0.25,0.18$, and $1.41 \mathrm{~ms}$ for I, III, and V, respectively, as gestational age increased.

Paludetti, et al. [5] studied 59 children diagnosed as normal. The authors observed age-related variations of the various ABR parameters due to the maturational development of auditory pathways and compared these with normal adult values. The children were divided into four age groups (3641 weeks gestational age, 1-6 months, 6-12 months, and $12-$ 36 months). The latency value of wave $\mathrm{V}$ decreased with increasing age, with decreased latency between groups. Change in wave $\mathrm{V}$ latency of $9.9 \mathrm{~ms}$ at 26 weeks of gestation to 6.9 $\mathrm{ms}$ at 40 weeks of gestation [6] and from $7.9 \mathrm{~ms}$ at $33-34$ weeks gestational age to $7.65 \mathrm{~ms}$ at $39-40$ weeks of gestational age [7]. A study of auditory brainstem responses in 535 children 3 months to 3 years of age reported that wave V latency decreased with age until 18 months of age, with little or no change noted in wave I latency at the same ages [8]. Inter-peak latency differences followed the same developmental time period as wave V. Another study that addressed normative brainstem auditory evoked potentials in children reported increasingly higher absolute latency and inter-peak latency with lower age [7]. Elsewhere, measurements of brainstem auditory evoked response in normal term neonates indicated mean latencies of wave I (1.79), II (2.88), III (4.54),
IV (5.86), V (6.75) and inter-peak latency I-III (2.75), III-V (2.22), and I-V (4.97) [9]. The authors observed decreased latency with increasing age. A marked discrepancy of wave $\mathrm{V}$ latency and inter-peak latency was noted.

In addition, Table 1 Summarizes the different methods used in difference studies and highlights the lack of information from infancy to childhood.

The present study is based on the assumption that the development of the brain is predominantly sensitive to auditory maturation, and that the development of auditory pathways during sensitive periods in early childhood ensures normal hearing.

\section{Need for the study}

Age is a very important factor in the diagnosis of ABR waveforms, especially for wave $\mathrm{V}$ as it is a prominent and reliable parameter to establish the threshold of hearing. The value of the peak latency varies among different age groups. The effect of maturation is age-dependent and differs from infancy to childhood. Limited literature is available concerning the effect of maturation across age in India and world-wide. The significance of this study is that it includes age groups from infants to children (birth to 12 years), which provide a more accurate maturation pattern of latency of wave $\mathrm{V}$ in infancy and beyond. The present study was undertaken to understand the effect of age on auditory maturation with age from infancy.

\section{Objective}

To assess the effect of age-related auditory maturation on ABR wave $V$ latency with age

\section{Subjects and Methods}

After obtaining approval from the institutional ethics com-

Table 1. Summary of previous studies

\begin{tabular}{|c|c|c|c|}
\hline Authors & Age-range & Wave V latency (ms) & Methodology \\
\hline Sleifer, et al. [10] & $4,12 \& 20$ months & $6.90,6.60,6.11$ & $\begin{array}{l}\text { ABR-click stimuli, TDH } 49 \text { headphone, low pass 1,500 Hz } \\
\text { \& high pass } 100 \mathrm{~Hz} \text {, rarefaction, 2,000 stimuli, analysis } \\
\text { window } 10 \mathrm{~ms}\end{array}$ \\
\hline Werner, et al. [11] & 3, 6 months, adults & $8.99,8.82,8.43$ & $\begin{array}{l}\text { ABR-tone pips stimuli, insert earphone, rate 13.3/s, 2,048 } \\
\text { stimuli }\end{array}$ \\
\hline Guilhoto, et al. [9] & Newborn (0-1 mont), adults & $6.75,5.6$ & $\begin{array}{l}\text { ABR-2,000 clicks, Intensity } 80 \mathrm{dBHL} \text {, alternating polarity, } \\
\text { rate } 10 / \mathrm{s} \text {, analysis time } 10 \mathrm{~ms} \text {, filter setting } 100-3 \mathrm{KHz}\end{array}$ \\
\hline Spitzer, et al. [12] & 3,5 years & $5.76,5.57$ & $\begin{array}{l}\text { ABR-2,000 clicks, insert earphone, intensity } 70 \mathrm{dBnHL} \text {, } \\
\text { polarity rarefaction, rate } 31 / \mathrm{s} \text {, response recorded } \\
\text { during movie watching, electrode impedance } 3 \mathrm{k} \Omega \text {, } \\
\text { filter settings } 100-1,500 \mathrm{~Hz} \text {, analysis time } 10 \mathrm{~ms}\end{array}$ \\
\hline Norrix, et al. [13] & 30-33 months, 19 years & $5.95,5.65$ & $\begin{array}{l}\text { ABR-clicks, intensity } 80 \mathrm{dBnHL} \text {, circum-aural headphone, } \\
\text { polarity rarefaction, rate } 13.3 / \mathrm{s}\end{array}$ \\
\hline
\end{tabular}

ABR: auditory brainstem response 
Table 2. Subject groups by age

\begin{tabular}{ccccccccc}
\hline Group & A & B & C & D & E & F & G & H \\
\hline Age range, months & $0-6$ & $7-12$ & $13-24$ & $25-36$ & $37-48$ & $49-60$ & $61-84$ & $85-144$ \\
\hline
\end{tabular}

mittee and informed consent from the parents or caregivers all the test procedures were performed.

\section{Participants}

The study involved 80 subjects ranging in age from newborn to 12 years. The age range was based on the electrophysiological changes that occur during maturation of the auditory pathway [14]. In the latter study, maturation of cortical axons until 12 years of age was demonstrated. Maturation of the brainstem and pathways are later followed by maturation of thalamocortical and intracortical connections [1]. However, it is possible that the specific lemniscal \& extralemniscal auditory pathways mature with different rates. The maturation of the peripheral \& cortical structure suggests human auditory maturation can be divided into several periods based on structural or functional temporal landmarks. Anatomical development into several period discover the separation of the maturational sequence [1].

Presently, the 80 subjects were allocated to eight age groups of 10 subjects each (Table 2). Subjects with history or risk of hearing loss, ear infection, neurological disorder, learning difficulty, or attention difficulty were excluded. All the subjects underwent an otoscopic examination to rule out any infection or middle ear pathology. Behavioral observation audiometry was done to see the minimum response level and diagnostic otoacoustic emission was administered to check the functioning of outer hair cells. Subjects with normal function of outer hair cells were selected.

\section{Stimulus and recording parameters}

ABR were elicited by an acoustic click. Brainstem responses collected in the same manner and during the same recording session. A Neurosoft device (Neuro-audio net-1.0.98.0) was used to determine ABR. Silicon wired gold plated cup electrodes was used during ABR testing. A click is a brief square wave with broad spectral content. For the click-evoked response, the stimuli were 2,000 presented at a rate of 21.1 stimuli per s. Stimuli were presented at $30 \mathrm{dBnHL}$ and 0.1 $\mathrm{ms}$ rectangular pulse trains through insert earphones to each ear separately (3A Insert Earphone, Ear Tone). Insert earphones were used as it prevents ear canal collapse, increases interaural attenuation, and are comfortable for a longer period of time, which may assist in reducing patient artifacts and can more effectively attenuate surrounding environmental noise. Stimulus intensity was calibrated in $\mathrm{dBnHL}$; amplitude and stimulus rate were calibrated using a dual trace oscilloscope (Hewlett Packard, Palo Alto, CA, USA) at the beginning and end of each test session. Two channel recording was done to measure the responses. This allowed for a comparison of the response recorded in each channel to determine the responsive ear. Filter settings were $30 \mathrm{~Hz}$ on the low-frequency end (high-pass setting) and 3,000 $\mathrm{Hz}$ on the high-frequency end (low-pass setting) with an analysis window of $12 \mathrm{~ms}$. Decreasing the high-pass filter settings emphasized wave V. The electrode placement for two-channel recording was based on the International 10-20 system, which is the most commonly used system. In this system, the vertex electrode position is $\mathrm{Cz}$, left ear is $\mathrm{A} 1$, the right ear is $\mathrm{A} 2$, and the forehead is Fpz with a contact impedance of $<5 \mathrm{k} \Omega$ for all electrodes, and fairly equal impedance within about 2,000 ohms, which efficiently minimizes background interference. Electrodes were first applied by cleaning the surface of skin with Nuprep gel and then placing a conductive electrode gel on the skin and electrode. The electrode was attached with tape. Electrode impedance was checked after the earphones and the patient was positioned for testing and also during testing, because changes in impedance may affect the quality of the recordings. The sampling rate was $40,000 \mathrm{~Hz}$ and responses was an online band passed filtered, waveforms were averaged online. The recording window was $1.5 \mathrm{~ms}$ starting after the stimulus onset. Here, the ABR was obtained with alternatively rarefaction and condensation clicks.

\section{Analysis}

Click-evoked response and peak latency of wave $\mathrm{V}$ was identified for each subject. The test was administered by a qualified and experienced audiologist and the wave identification was done by four audiologists. Wave $\mathrm{V}$ was identified at the point on the waveform before the negative slope that follows the wave. Contralateral recording was done, which helped to identify wave V. Consistency in wave identification and reliability was ensured.

\section{Results}

The absolute latency of wave $\mathrm{V}$ of click-evoked ABR was measured. Wave $\mathrm{V}$ was present in all the participants. Table 3 shows mean and standard deviation of latency values of wave $\mathrm{V}$ for each ear. One way ANOVA was done to examine the effect of age on wave $V$ latency of ABR across groups. 
Table 3. Mean and SD of latency values of wave $V$ for the right and left ears

\begin{tabular}{ccccccc}
\hline \multirow{2}{*}{ Group } & $\begin{array}{c}\text { Age range, } \\
\text { months }\end{array}$ & \multicolumn{2}{c}{ Right } & & \multicolumn{2}{c}{ Left } \\
\cline { 3 - 4 } \cline { 6 - 7 } & Mean & SD & & Mean & SD \\
\hline A & $0-6$ & 8.16 & 0.52 & & 8.32 & 0.49 \\
B & $7-12$ & 7.67 & 0.59 & & 7.67 & 0.54 \\
C & $13-24$ & 7.56 & 0.40 & & 7.58 & 0.55 \\
D & $25-36$ & 6.60 & 0.31 & & 6.58 & 0.25 \\
E & $37-48$ & 6.15 & 0.12 & & 6.15 & 0.15 \\
F & $49-60$ & 5.99 & 0.14 & & 5.99 & 0.12 \\
G & $61-84$ & 5.74 & 0.11 & & 5.75 & 0.11 \\
H & $85-144$ & 5.69 & 0.13 & & 5.65 & 0.11 \\
\hline SD: standard deviation & & & &
\end{tabular}

Latency of the wave $\mathrm{V}$ decreases very rapidly until 3 years of age, after which the maturation of wave $\mathrm{V}$ becames slower and was complete by 12 years of age. The absolute latency of wave $\mathrm{V}$ occurred within the first $8-9 \mathrm{~ms}$ in early age. The interaural latency difference in children was $0.2 \mathrm{~ms}$. There was no significant difference in latency of wave $\mathrm{V}$ between the ears among all the age groups.

Post-hoc analysis was done to see the age effect between the groups in both ears. There was a significant decrease in latency between groups until 3 years of age, but there was no significant decrease in wave $\mathrm{V}$ latency after 3 years between groups. This indicated that maturation of the wave $\mathrm{V}$ occurs until 3 years of age and then became stable. Auditory maturation slowed between 1 to 2 years of age, perhaps due to the growth of axons. The absolute latency of wave $\mathrm{V}$ decreased very rapidly in all groups from 3 years of age. This suggests that auditory maturation is faster earlier in life. At the age of 3-4 years, maturation slowed but continued until 12 years of age.

\section{Discussion}

Development of the brain leads to auditory maturation, with the central auditory system maturing with age. ABR is one of the qualitative and quantitative measures to see the maturation processes in children. The aim of this study was to assess the age-related changes or auditory maturation on ABR wave V latency. The findings will help clarify the development of the auditory pathway from infancy to childhood. Some studies suggest that auditory pathways mature until up to 12 years of age [10], whereas others describe maturation at 2-3 years of age [4]. The present study selected an age range of infancy to 12 years of age in order to provide clarity to the contrasting findings. The subjects formed eight age groups on the assumption that auditory maturation could take place in several age periods, based on structural and func- tional landmarks.

The latency of ABR wave $\mathrm{V}$ regularly changed during the first 3 years of life. The decrease in absolute latency of ABR could be due to rapid increase in axonal myelin density in the cochlear nerve and brainstem pathways [1]. Adult-like maturity of wave $\mathrm{V}$ by 2 years of age has been described [1]. Wave $\mathrm{V}$ occurred initially within the first $8-9 \mathrm{~ms}$ following the onset of the stimulus. This could be because myelinization of the inferior colliculus takes place at the age of 1 year, though fine-tuning takes place later during the second year of life and the density of myelin increases with age, with functional maturity by 3 years of age. These neural responses likely occur within the inferior colliculus [4]. Maturation of the primary auditory nervous system could conceivably play a part. Synaptic immaturity could lead to loss of information along the neural pathways, which would in turn lead to reduced sensitivity, prolonged evoked potential latency, and reduced evoked potential amplitude in young children. The primary auditory pathways central to the brainstem may continue to mature beyond 6 months of life [15]. The observed inter-aural latency difference of $0.2 \mathrm{~ms}$ indicates that the development of the auditory pathway to the inferior colliculus occurs simultaneously on both sides.

The present findings are limited by the limited number of participants in each group.

\section{Conclusion}

It is clear that there is a distinct maturational pattern for ABR. Changes in ABR latency of wave V suggests involvement of both peripheral and central auditory structures. The present study reveals infant's response mature at 3 years of age, whereas absolute latency values continuously develop, even after from peripheral to central, and reach the adult value between 6 to 12 years of age. Birth to 3 years of age is the critical period for the development of the auditory system. ABR is a reliable test to assess maturational changes of auditory and brain stem function. Myelinization of the auditory nerve and brainstem pathways is faster in the early age of life which enables children to learn faster. There was a significant difference in wave $\mathrm{V}$ latency among all the groups until 3 years of age, with no significant difference after 3 years of age among the groups. This could reflect the stabilization of cortical density from 2 to 5 years of age [10]. This results support the conclusion that anatomical maturation of the auditory system is manifest as progressive changes in ABR and supports the prior finding that the period from birth to 12 years of age is characterized by major transient changes in cortical evoked potential [16]. 


\section{Acknowledgments}

The authors would like to thank to the institutional research committee for giving the opportunity to conduct this study. This article is a part of a research project, funded by SRH University, Jollygrant, Dehradun.

\section{Conflicts of interest}

The authors have no financial conflicts of interest.

\section{REFERENCES}

1) Johnson KL, Nicol T, Zecker SG, Kraus N. Developmental plasticity in the human auditory brainstem. J Neurosci 2008;28:4000-7.

2) Sininger YS, Abdala C, Cone-Wesson B. Auditory threshold sensitivity of the human neonate as measured by the auditory brainstem response. Hear Res 1997;104:27-38.

3) Weber BA. Comparison of auditory brain stem response latency norms for premature infants. Ear Hear 1982;3:257-62.

4) Jacobson JT, Morehouse CR, Johnson MJ. Strategies for infant auditory brain stem response assessment. Ear Hear 1982;3:263-70.

5) Paludetti G, Maurizi M, Ottaviani F, Rosignoli M. Reference values and characteristics of brain stem audiometry in neonates and children. Scand Audiol 1981;10:177-86.

6) Kaga K, Hashira S, Marsh RR. Auditory brainstem responses and behavioural responses in pre-term infants. Br J Audiol 1986;20:121-7.

7) Scaioli V, Brinciotti M, Di Capua M, Lori S, Janes A, Pastorino G, et al. A Multicentre Database for Normative Brainstem Auditory Evoked Potentials (BAEPs) in Children: Methodology for Data Collection and Evaluation. Open Neurol J 2009;3:72-84.
8) Gorga MP, Kaminski JR, Beauchaine KL, Jesteadt W, Neely ST. Auditory brainstem responses from children three months to three years of age: normal patterns of response. II. J Speech Hear Res 1989; 32:281-8.

9) Guilhoto LM, Quintal VS, da Costa MT. Brainstem auditory evoked response in normal term neonates. Arq Neuropsiquiatr 2003;61: 906-8.

10) Sleifer P, da Costa SS, Cóser PL, Goldani MZ, Dornelles C, Weiss $\mathrm{K}$. Auditory brainstem response in premature and full-term children. Int J Pediatr Otorhinolaryngol 2007;71:1449-56.

11) Werner LA, Folsom RC, Mancl LR. The relationship between auditory brainstem response latencies and behavioral thresholds in normal hearing infants and adults. Hear Res 1994;77:88-98.

12) Spitzer E, White-Schwoch T, Carr KW, Skoe E, Kraus N. Continued maturation of the click-evoked auditory brainstem response inpreschoolers. J Am Acad Audiol 2015;26:30-5.

13) Norrix LW, Trepanier S, Atlas M, Kim D. The auditory brainstem response: latencies obtained in children while under general anesthesia. J Am Acad Audiol 2012;23:57-63.

14) Ivone FN, Schochat E. [Auditory processing maturation in children with and without learning difficulties]. Pro Fono 2005;17:311-20.

15) Moore JK, Perazzo LM, Braun A. Time course of axonal myelination in the human brainstem auditory pathway. Hear Res 1995;87: 21-31.

16) Roncagliolo M, Benítez J, Eguibar JR. Progressive deterioration of central components of auditory brainstem responses during postnatal development of the myelin mutant taiep rat. Audiol Neurootol 2000;5:267-75. 